\title{
Mapeamento Sistemático Sobre o Uso de Tecnologias para o Aprendizado de Processos Químicos
}

\section{Introdução}

A química tem uma grande participação nos dias atuais com os inúmeros produtos fundamentais à humanidade. A sua presença pode ser destacada desde diversos combustíveis aos mais complexos medicamentos ${ }^{1}$. Porém, a produção química também gera inúmeros inconvenientes, como a formação de subprodutos tóxicos e a contaminação do ambiente e do próprio homem expostos a estes xenobióticos ${ }^{2}$.

A preocupação com estes inconvenientes pode ser claramente observada pois, nos últimos anos, cresce continuamente a pressão sobre as indústrias químicas, tanto por meio da sociedade civil como das autoridades governamentais, no sentido de aprimorar o desenvolvimento de processos, que sejam cada vez menos prejudiciais ao meio ambiente ${ }^{3}$.

Dentro da problemática industrial vigente, um dos principais problemas que se destaca é o grande volume de efluentes tóxicos produzidos por vários processos químicos ${ }^{4}$. A emissão de contaminantes pode ser minimizada através de diversos caminhos, tais como o emprego de reagentes alternativos apropriados ${ }^{5}$; o aumento da seletividade para maximizar o uso dos materiais de partida ${ }^{6}$; a utilização de catalisadores para facilitar a separação do produto final da mistura; a reciclagem dos reagentes e catalisadores empregados no processo ${ }^{6-8}$.

Dentre as áreas de pesquisa focadas para estas finalidades, tem se destacado muito nos últimos anos a preparação de catalisadores sólidos, com o firme propósito da remoção de contaminantes dispersos em efluentes, bem como na catálise de reações químicas com o objetivo da maximização das reações e redução da formação de subprodutos indesejáveis durante o processo reacional ${ }^{9,10}$.
Estes conceitos devem estar fixados em todos os estudantes de química com a intenção formar profissionais capacitados para os novos conceitos científicos e tecnológicos responsáveis pela sustentabilidade do planeta ${ }^{11,12}$.

Neste contexto, o objetivo deste trabalho é verificar o panorama das pesquisas relacionadas sobre o uso de tecnologias para aprendizado de processos químicos, apresentando um estudo sistêmico acerca do que vem sendo publicado para tal vertente. A finalidade deste mapeamento sistemático é analisar as referências dos trabalhos realizados envolvendo a temática sobre as ferramentas tecnológicas utilizadas para aprendizado de processos químicos. E, com isso, verificar a ascensão desta linha de pesquisa na atualidade.

\section{Metodologia}

A fim de efetuar-se a revisão da literatura estabelecida pelo trabalho, um mapeamento sistemático (mapping study) foi realizado de acordo com a metodologia proposta ${ }^{13}$ e 14 , que consiste em uma busca de estudos cadastrados em bases de dados por meio de operadores lógicos para a seleção dos artigos, a partir da seleção de palavras ou expressões chave.

As bases de dados consideradas para o presente trabalho foram: IEEE Xplore ${ }^{15}$, Science Direct ${ }^{16}$, Scielo ${ }^{17}$, Scopus ${ }^{18}$ e Periódicos $\mathrm{CAPES}^{19}$, sendo estas bases disponíveis na Universidade Federal de Catalão. Faz-se necessário ressaltar que foram analisados somente artigos de periódicos revisados por pares.

As expressões lógicas utilizadas para a busca nas bases foram: "technology" AND "learning" AND "chemical processes". Foram escolhidas estas strings para buscar trabalhos que envolvam tecnologias para aprendizado de processos químicos. Em seguida, foram aplicados filtros 
para reduzir o escopo da busca. Por exemplo, quanto ao idioma (inglês e português), tipo de publicação (artigo de periódico revisado por pares) e ano de publicação (de 2013 a 2019, constituindo 7 anos de espaço de busca). Aplicados os filtros em cada base de dados, foi feita uma leitura dos títulos de forma a selecionar quais estavam de acordo com a expressão lógica selecionada. Observou-se também as possíveis repetições entre as bases de dados, e os artigos que se encaixavam nos critérios de inclusão tiveram quaisquer duplicatas removidas.

Por fim, a etapa final de seleção dos artigos consistiu no direcionamento para aplicações que envolviam tecnologias para aprendizado de processos químicos. Para tanto, foi efetuada a partir da leitura e análise dos títulos e resumos, de modo a excluir aqueles trabalhos que não se relacionavam diretamente ao tema em estudo, sendo a revisão da bibliografia desenvolvida a partir deste resultado obtido. Neste sentido, trabalhos que abordavam temáticas diferentes foram descartados, como trabalhos que abordavam tecnologias para aprendizado voltados para aplicação em outras áreas, por exemplo.

\section{Discussão e Resultados}

Após a realização das buscas nas bases de dados, os resultados obtidos foram organizados em forma de tabelas e gráficos com a finalidade de apresentá-los de maneira mais prática. A Tabela 1 apresenta os resultados totais obtidos no mapeamento, considerando-se as citadas palavras-chave e metodologias citadas na seção anterior.

Dessa forma, por meio do mapeamento sistemático, foi criado um banco de dados com 35 (trinta e cinco) referências de bases de dados para a combinação de "technology" AND "learning" AND "chemical processes".

A evolução da publicação anual dos trabalhos selecionados na busca internacional pode ser vista na Figura 1, na qual se nota que os artigos envolvendo o assunto abordado neste trabalho foram publicados nos últimos anos. Dessa forma, observa-se que o assunto é recente e com grandes possibilidades de campos a serem explorados.
Tabela 1. Síntese dos resultados obtidos nas pesquisas.

\begin{tabular}{|c|c|c|}
\hline \multirow{2}{*}{ Banco de dados } & \multicolumn{2}{|c|}{ Número de artigos } \\
\cline { 2 - 3 } & Inicial & Com título aderente \\
\hline IEEE Xplore & 5 & 4 \\
\hline Science Direct & 53 & 9 \\
\hline Scielo & 30 & 8 \\
\hline Scopus & 18 & 5 \\
\hline Periódicos CAPES & 41 & 10 \\
\hline Total & 147 & 35 \\
\hline
\end{tabular}

\section{Número de artigos por ano de publicação}

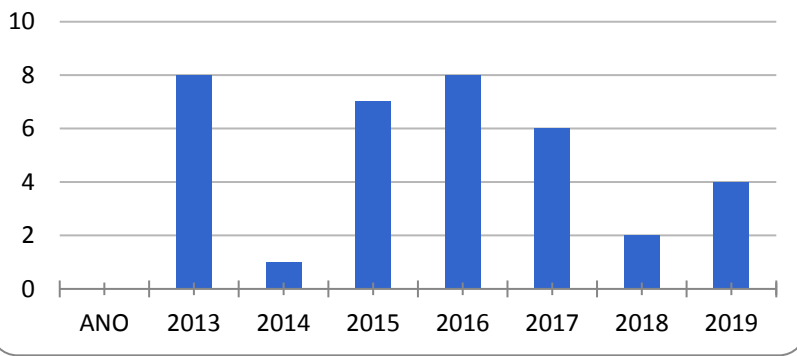

Figura 1. Número de artigos por ano de publicação.

Os 35 (trinta e cinco) trabalhos selecionados constam nas referências do presente trabalho. Porém, não estão citados no corpo do texto devido à extensa quantidade.

A Figura 2 apresenta o número de artigos de periódicos publicados por países. Os trabalhos publicados são de diferentes países, tais como: Argentina, Austrália, Bangladesh, Brasil, Chile, China, Coréia do Sul, Espanha, Estados Unidos, França, Índia, Indonésia, Itália, Japão, Nova Zelândia e Romênia. Desse modo, verifica-se que a maioria dos trabalhos encontrados que englobam o uso de tecnologias para aprendizado de processos químicos são oriundos de países desenvolvidos, inclusive foi encontrado apenas um trabalho que aborda esse tema no Brasil.

A Figura 3 exibe a quantidade de artigos selecionada de acordo com a tecnologia utilizada para aprendizado de processos químicos, as quais foram variadas, dentre as quais 
citam-se: algoritmo Adaboost detecção facial; customizado; detecção de Blob; detecção de cores com OpenCV; detecção de gestos e objetos; detecção facial e angulação facial; fuzzy controler e realidade virtual; HOG (Histogram of Oriented Gradients) com OpenCV; modelo cinemático e movimento da íris; odometria visual monocular; OpenCV, OpenNI e reconhecimento de gestos; PCl (Point Cloud Library); RV (virtual reality) e FOV (field of view); SLAM (Simultaneous Localization and Mapping) baseado em visão; TensorFlow; visão computacional egocêntrica; visão estéreo; visão monocular; detecção e mapeamento de obstáculos. A maioria dos trabalhos utilizou ferramenta customizada, ou seja, as tecnologias utilizadas foram adaptadas segundo à necessidade dos pacientes.

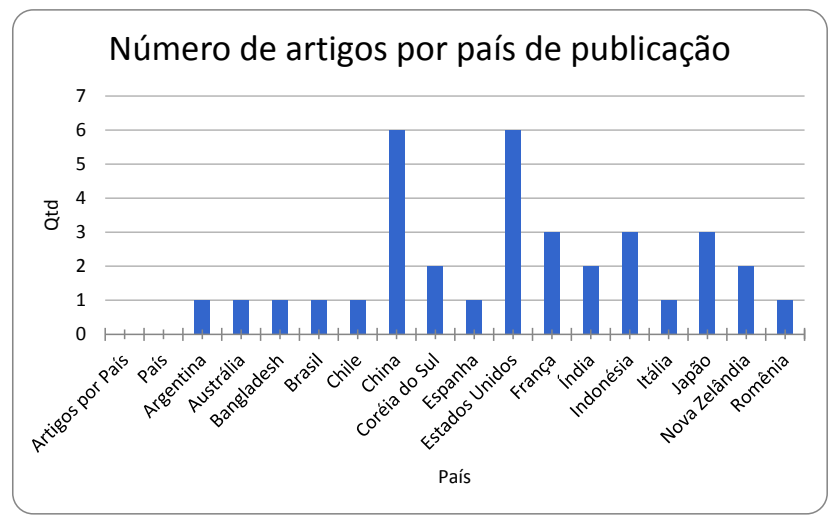

Figura 2. Número de artigos por país de publicação.

A Figura 4 apresenta a quantidade de artigos encontrada conforme o tipo de equipamento utilizado nos trabalhos sobre uso de tecnologias para aprendizado de processos químicos, a saber: câmera; câmera binocular e emotiv epoc; câmera de visão estéreo; câmera e braço robótico; câmera e laser de linha; câmera e led infravermelho; câmera e raspberry pi; câmera e sensor IMU; câmera e LIDARLite; câmera, raspberry pi e laser de linha; câmera e tablet; infravermelhos e ultrassons; Kinect e Tobii PCEye; simulador e câmera; ultrassons; Xtion PRO Live. Dessa forma, percebe-se que a câmera foi o equipamento mais utilizado nos trabalhos selecionados a respeito da temática pesquisada neste mapeamento sistemático.

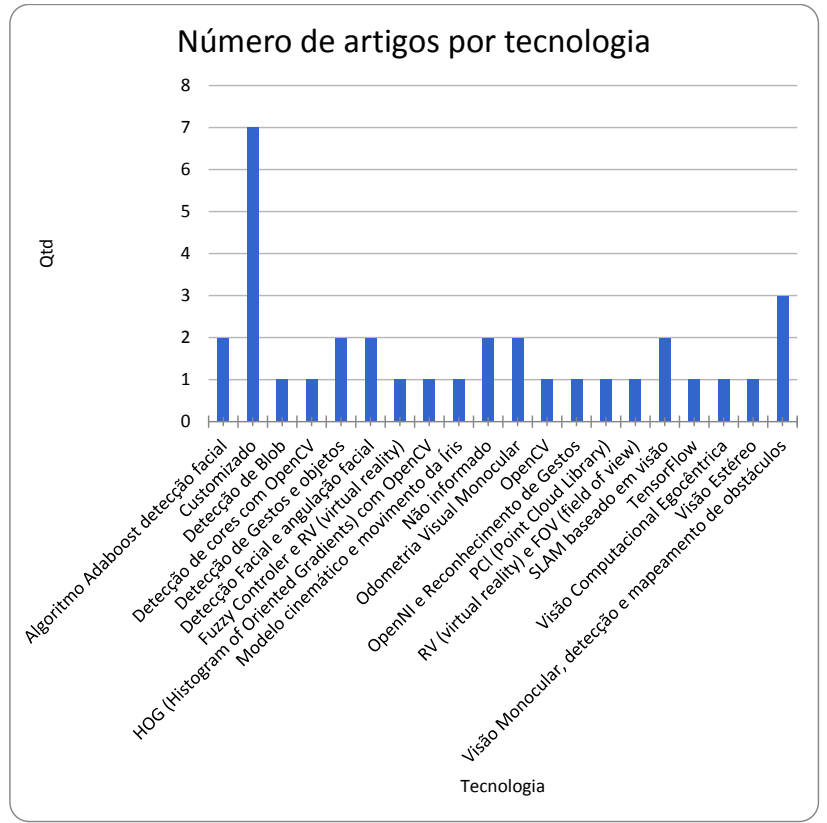

Figura 3. Número de artigos por tecnologia.

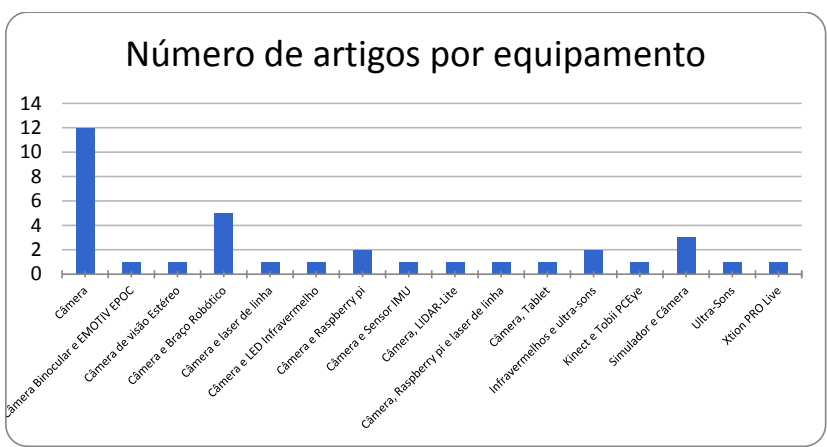

Figura 4. Número de artigos por equipamento.

A Figura 5 apresenta a porcentagem de artigos que fizeram experimentos com participantes. Dessa forma, observa-se que a maioria (69\% dos trabalhos) realizou este procedimento. Isso demonstra a necessidade da realização de testes para comprovação dos resultados e da eficácia das aplicações, além de auxiliar na realização de melhorias e manutenção dos sistemas.

\section{Conclusões}

Por meio do mapeamento realizado, foi possível verificar que houve um crescimento no estudo da aplicação do uso 
de tecnologias para aprendizado de processos químicos, visto que é uma técnica nova e muito eficiente.

\section{Porcentagem de artigos que fizeram experimentos}

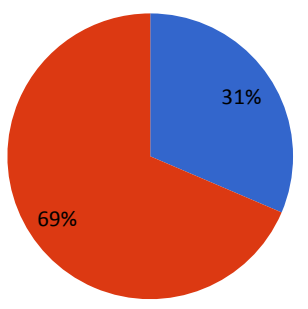

Não mencionado - Sim

Figura 5. Porcentagem de artigos que fizeram experimentos.

Assim, cabe destacar que a aplicação do mapeamento sistemático na elaboração de revisão bibliográfica permite identificar as principais lacunas para o desenvolvimento de novas pesquisas. Além disso, direciona para as principais publicações ligadas ao estudo.

Em virtude do que foi apresentado neste trabalho, verifica-se que há um crescente interesse em pesquisar e publicar nesta área relacionada ao uso de tecnologias para aprendizado de processos químicos, procurando auxiliar na formação de pessoas desta área.

Portanto, percebe-se a necessidade de fomentar esta área de pesquisa para oferecer a este público às tecnologias como forma de treinamento, aquisição de conhecimento, motivação, entretenimento ou mesmo por inclusão. Dessa maneira, poderá se obter uma maior maturidade nos resultados obtidos e, assim, promover uma sistematização no emprego de tecnologias para processos químicos no auxílio da promoção do bem-estar destas pessoas.

\section{Referências}

1. Poliakoff, M.; Fitzpatrick, J. M.; Farren, T. R.; Anastas, P. T.; Science 2002, 297, 807 .

2. Graedel, T.; Green Chem. 1999, 1, G126.

3. Clark, J. H.; Green Chem. 1999, 1, 1 .
4. Tundo, P.; Anastas, P.; Black, D. S.; Breen, J.; Collins, T.; Memoli, S.; Myiamoto, J.; Polyakoff, M.; Tumas, W.; Pure Appl. Chem. 2000, $72,1207$.

5. Clark, J. H.; Macquarrie, D. J.; Chem. Commun. 1998, 853.

6. Clark, J. H.; Macquarrie, D. J.; Chem. Soc. Rev. 1996, 303.

7. Price, P. M.; Clark, J. H.; Macquarrie, D. J.; J. Chem. Soc., Dalton Trans. 2000, 101.

8. Anatas, P. T.; Kirchhoff, M. M.; Acc. Chem. Res. 2002, 35, 686.

9. Bresson, C.; Menu, M. J.; Dartiguenave, M.; Dartiguenave, Y.; J. Environ. Monit. 2000, 2, 240.

10. Stein, A.; Melde, B. J.; Schoden, R. C.; Adv. Mater. 2000, 12, 1403.

11. Hjeresen, D. L.; Schutt, D. L.; Boese, J. M.; J. Chem. Educ. 2000, $77,1543$.

12. Matlack, A.; Green Chem. 1999, 1, G19.

13. Bailey, J. et al.. Evidence relating to Object-Oriented software design: A survey. First International Symposium on Empirical Software Engineering and Measurement. Computer Society, 2007.

14. Petersen, K. et al. Systematic Mapping Studies in Software Engineering. School of Engineering, Blekinge Institute of Technology. University of Bari, Italy, 26 - 27 June. 2008.

15. . IEEE Xplore Digital Library. 2019. Disponível em: $<\mathrm{https}: / /$ ieeexplore.ieee.org>. Acesso em: 18 set. 2019.

16. Science Direct. 2019. Disponível em: $<$ http://www. sciencedirect.com/>. Acesso em: 18 set. 2019.

17. __ Scielo. 2019. Disponível em: $<$ https://scielo.org/>. Acesso em: 18 set. 2019.

18. _. Scopus. 2019. Disponível em: $<$ https://www.scopus.com/>. Acesso em: 18 set. 2019

19. _. CAPES - Portal de Periódicos da Coordenação de Aperfeiçoamento de Pessoal de Nível Superior. 2019. Disponível em: < http://www-periodicos-capes-gov-br.ez49.periodicos.capes. gov.br/>. Acesso em: 18 set. 2019

\section{Flávia G. Fernandes* \& Marcos N. Rabelo}

\author{
Programa de Pós-Graduação em Ciências Exatas e Tecnológicas \\ Universidade Federal de Catalão, \\ *E-mail: flavia.fernandes92@gmail.com
}


